\title{
Vaginal mesh vs. native tissue repairs for prolapse: FDA update and recent evidence
}

\author{
Catherine S. Bradley, MD, MSCE${ }^{1}$
}

In 2010, 300,000 surgeries were performed in the U.S. for pelvic organ prolapse (POP), and 100,000 of those involved the placement of surgical mesh. The use of mesh in abdominal surgeries for POP has been described for over 50 years, but the first surgical mesh product specifically for POP was approved by the FDA in 2002. Over the last decade, transvaginal mesh POP surgeries have become more common, and mesh "kits" have been approved and are increasingly used for transvaginal POP repair, but clinical trial evidence of their safety and effectiveness has lagged behind rapid adoption into practice.

Mesh use has been advocated in part because of low success rates after traditional vaginal POP repairs. However, increasing research suggests previously recommended anatomic outcomes for prolapse surgery are too strict, as these definitions would classify many "normal" women as having prolapse. Furthermore, evidence shows that subjective symptom outcomes that include an absence of bulge symptoms correlate best with patients' impression of improvement, while outcomes based solely on anatomic criteria do not. Recent recommendations are that POP surgery definitions of success should include the absence of bulge symptoms in addition to anatomic criteria. Reanalysis of trial data using such outcomes demonstrate high success rates $(>80 \%)$ for traditional vaginal repairs (e.g. anterior colporrhaphy), in contrast to lower success rates based on strict anatomic criteria alone.

In July 2011 the FDA published an "Update on Safety and Effectiveness for Transvaginal Placement of Surgical Mesh for Pelvic Organ Prolapse," summarizing recent Manufacturer and User Device Experience (MAUDE) database reports related to transvaginal mesh repairs and results of a systematic literature review. From 2008-2010, 1503 MAUDE reports associated with mesh for POP were entered, a 5-fold increase compared to the previous 3-year period. Complications frequently reported included mesh erosion, pain/dyspareunia, infection, urinary problems, bleeding and organ perforation. A systematic review of over

${ }^{1}$ Department of Obstetrics and Gynecology, The University of lowa, lowa City, IA, 52240

Please cite this paper as: Bradley CS. Vaginal mesh vs. native tissue repairs for prolapsed: FDA update and recent evidence. Proc Obstet Gynecol. 2011 November;2(2):Article 10 [ 2 p.]. Available from: http://ir.uiowa.edu/pog/. Free full text article.

Corresponding author: Catherine S. Bradley, Department of Obstetrics and Gynecology, University of lowa, 51217 PFP, 200 Hawkins Drive, lowa City, IA, 52242. Telephone (319)356-1534, catherine-bradley@uiowa.edu

This is an Open Access article distributed under the terms of the Creative Commons Attribution 3.0 Unported License (http://creativecommons.org/licenses/by/3.0), which permits unrestricted use, distribution, and reproduction in any medium, provided the original work is properly cited. 
11,000 women from 110 studies of transvaginal mesh repairs found then overall mesh erosion incidence was $10.3 \%$, and more than half of cases required surgical intervention.

The FDA also reviewed evidence for the efficacy of transvaginal mesh repairs, concluding that clinical benefit for apical and posterior mesh repairs over traditional repairs has not been demonstrated. Anterior vaginal mesh repair does appear to confer an anatomic benefit compared to non-mesh procedures, but this may not result in better outcomes. Thus, the current literature suggests that transvaginal mesh POP repairs expose patients to additional risks and do not conclusively improve overall outcomes compared to traditional repairs. The FDA is now considering regulatory changes to the approval process for transvaginal mesh devices, which might include an increase in risk classification, which would require manufacturers to present clinical data before approval, as well as expanded post-market monitoring or surveillance requirements for currentlyapproved devices.

In this uncertain climate surrounding the use of transvaginal mesh, clinicians should be familiar with the available evidence, make informed decisions about recommended procedures and tailor choice of procedure to each patient. Sufficient surgeon training and volume in mesh POP surgeries are important to optimize outcomes. Most importantly, surgeons must ensure that patients understand the risks and benefits related to mesh through a thorough and well-documented informed consent process.

Vaginal mesh vs. native tissue repairs for prolapse 